\title{
An overview of pregnancy and fertility issues in breast cancer patients
}

\author{
Charlotta Dabrosin
}

\section{Linköping University Post Print}

\section{Tweet}

N.B.: When citing this work, cite the original article.

This is an electronic version of an article published in:

Charlotta Dabrosin, An overview of pregnancy and fertility issues in breast cancer patients, 2015, Annals of Medicine, (47), 8, 673-678.

Annals of Medicine is available online at informaworldTM:

http://dx.doi.org/10.3109/07853890.2015.1096953

Copyright: Taylor \& Francis: STM, Behavioural Science and Public Health Titles http://www.tandf.co.uk/journals/default.asp

Postprint available at: Linköping University Electronic Press http://urn.kb.se/resolve?urn=urn:nbn:se:liu:diva-123841 
An overview of pregnancy and fertility issues in breast cancer patients

Charlotta Dabrosin

Department of Oncology and Department of Clinical and Experimental Medicine, Linköping University, Linköping, Sweden

Correspondence:

Charlotta Dabrosin, MD PhD

Professor of Oncology

Linköping University

Division of Oncology

SE-581 85 Linköping, Sweden

Phone: +46101038595

E-mail: charlotta.dabrosin@liu.se 


\begin{abstract}
Breast cancer is one of the most common malignancies of women in the reproductive years. In the Western world there is a trend towards delaying pregnancy to later in life and in combination with an increased incidence of breast cancer an increased number of women are diagnosed with breast cancer before they have completed their reproductive plans. In addition, breast cancer during pregnancy may affect an increased number of women as the childbearing years are delayed. The survival rate after breast cancer has improved during the last decades and many young breast cancer survivors will consider a pregnancy subsequent to the completion of adjuvant breast cancer therapy. Traditionally, many women are advised against a pregnancy due to a fear of increased risk of recurrence, especially women with estrogen receptor positive breast cancer. Due to feasibility issues, evidence from large prospective randomized trials are missing regarding the safety of pregnancy after breast cancer. Today guidelines are based on cohort studies and population-based registry evidence with its limitations. Overall, data suggest that pregnancy after breast cancer therapy is safe and the current evidence is summarized in this overview.
\end{abstract}

Keywords: breast cancer, mammary cancer, pregnancy, estrogen receptor, fertility 


\section{Key messages:}

- Despite the lack of high-level evidence all retrospective data report no unfavorable effect on breast cancer outcome of a subsequent pregnancy after adjuvant breast cancer therapy.

- There are no reasons for proscription in principle against pregnancy for women after breast cancer therapy.

- The risk of premature menopause, infertility, and the suitability of fertility preservation approaches need to be discussed in a multidisciplinary setting with all eligible women before start of cytotoxic therapies.

- Women with breast cancer during pregnancy should be given appropriate systemic treatment as closely as possible to general guidelines. 


\section{Introduction}

Breast cancer is the most common cancer among women in the Western world and the incidence is still increasing in many Western countries $(1,2)$. Although the median age of this disease is over 60 years approximately $20 \%$ of all cases affect women under the age of 49 years and $5 \%$ of affected women are diagnosed under the age of $40(1,2)$. Today, the majority of women with early stage breast cancer will survive their disease due to improved treatment options. In combination with the trend of delaying the childbearing years a growing number of patients need counseling regarding pregnancy after completion of adjuvant therapy or regarding fertility preservation before the start of therapy. As the childbearing years are delayed an increased the risk of breast cancer during pregnancy may be foreseen and will demand an increased knowledge of the management of these patients.

In younger women the risk of affecting fertility by chemotherapy may in fact influence their treatment choices with the risk of a suboptimal breast cancer treatment (3). Counseling regarding these issue are therefore needed before start of adjuvant therapy but less than half eligible women has been found to receive such counseling (4). In addition, the advancements of fertility treatments have further improved the possibility of pregnancy in these women. Here, the recent literature regarding fertility issues, pregnancies in relation to breast cancer treatment, and outcome are summarized and discussed.

Cited papers were found by a literature search using PubMed for articles published until August 2015. Additionally, relevant professional society guidelines were searched. The following key words were used: breast cancer, pregnancy, fertility, ovarian metastasis, childbirth, amenorrhea, chemotherapy, menopause, fertility preservation, pregnancy associated breast cancer, and statistics. Only relevant, English- language articles were included. 


\section{Breast cancer during pregnancy}

A breast cancer diagnosis during pregnancy has up to date been regarded as unfavorable. Pregnancy associated breast cancer (PABCa) is defined as breast cancer diagnosed during pregnancy or within 1 year of delivery and occurs approximately in 1 of 3,000 pregnancies. A general belief has been that PABCa is associated with worse outcome and poor survival. This is supported by a recent large meta-analysis of 30 studies, which showed an increased risk of death in PABCa compared with women with non-PABCa (5). After adjustment for age and stage the difference became less pronounced, especially regarding age (5). Diagnosis of breast cancer in the postpartum period was associated with significantly poorer outcome (pooled hazard ratio (pHR) 1.85; 95\% CI: 1.28-2.65) compared with diagnosis during pregnancy (pHR 1.29; 95\% CI: 0.74-2.24) (5). However, the data from the literature is inconsistent and several cohort studies have shown that after adjustment to prognostic factors, pregnancy per se does not affect the disease-free or overall survival (6-8). Similar overall survival of PABCa was found in a multicenter registry study where 311 women diagnosed with breast cancer during pregnancy were identified and compared with 865 non-pregnant women (6). After adjustments for age, stage, grade, hormone receptor status, HER-2 status, histology, type of chemotherapy, use of trastuzumab, radiotherapy, and hormone therapy no significant difference between the groups was detected (6). In another study of 75 women receiving chemotherapy during PABCa, matched for age and cancer stage with two nonpregnant patients, a better five-year overall survival (OS) was detected; 77\% (95\% CI: 63.9\%-86.4\%) for pregnant patients and $71 \%$ (95\% CI: $61.1 \%-78.3 \%$ ) for controls (7). However, a study of 65 patients where PABCa patients were stage-matched with two non-pregnant controls concluded a poorer disease free survival (DFS) of PABCa (HR 2.3; 95\% CI: 1.3-4.2) compared to the non-pregnant controls (9). As there are no data from randomized controlled studies, for obvious reasons, the level of evidence is, however, low. A delay in the diagnosis and the risk of less effective treatment or a delay of 
treatment during pregnancy may explain the reports of worse prognosis of PABCa. This is supported by the study where there was no difference in survival between women with PABCa receiving chemotherapy matched for age and stage with non-PABCa (7). This emphasizes the importance of giving appropriate systemic treatment to women with PABCa.

\section{Pregnancy after breast cancer treatment}

Before any consultation regarding fertility preservation before the start of adjuvant breast cancer treatment a discussion regarding the safety of a pregnancy after completed therapy needs to be addressed. Due to the nature of the subject no randomized controlled studies have been performed, hence the data to refer to are retrospective registry studies and meta-analyses. One major issue to take into consideration in these studies is the "healthy mom" effect (10). Despite matching for age and stage in the registries the women that became pregnant after breast cancer treatment may represent a group of women free of relapse and/or in general healthier compared to the non-pregnant women. Another issue is whether there is a difference in recurrence rate depending on hormone dependent breast cancer compared to other types of the disease. A recent multicenter retrospective study comprising of 333 pregnancies after breast cancer and 874 matched non-pregnant controls was powered to detect possible differences regarding to tumor type (11). In addition to estrogen receptor (ER) status the patients were matched for nodal status, adjuvant therapy, age, and year of diagnosis (11). HER-2 status was unknown in approximately $80 \%$ of all cases. In both the ER positive group and in the ER negative group no difference of disease free survival was detected after pregnancy compared to controls (11). In addition, in the ER negative group a significant increase in overall survival was detected whereas in the ER positive group no difference in survival between index cases and controls

was revealed (11). Regarding the timing of pregnancy after breast cancer diagnosis a surprisingly increase in the disease free survival was detected in patients who became pregnant 
within two years of diagnosis whereas no difference was found in the group that became pregnant more than two years after breast cancer diagnosis (11). The authors do, however, believe that this was a result of a selection bias rather than a true protective effect of an early pregnancy (11). A large retrospective population based registry study support the finding of the safety of pregnancy after breast cancer diagnosis. Over 10,000 Danish women with breast cancer under the age of 45 years were followed for over 95,000 person years (12). Of these women, 371 experienced a pregnancy after breast cancer treatment (12). No information on tumor biology was included in but age, stage, nodal status, and pregnancy history were included in the multivariate analysis (12). A significant reduced risk of dying was detected in the group of women who experienced a full-term pregnancy compared to the group of women that did not (RR 0.73 95\% CI: 0.54-0.99) (12). In addition, no negative effect on prognosis was detected after spontaneous or induced abortion (12). In another population based cancer registry in three different states in the US, 438 women having birth after breast cancer diagnosis were identified (8). 2775 women were matched for age, ethnicity, year of diagnosis, stage, and previous nonbreast primary tumors (8). Women giving birth 10 or more months after diagnosis were found to have a significant decrease risk of dying compared to those who did not (RR $0.5495 \% \mathrm{CI}$ : 0.41-0.71) (8). In the group of very young women, under the age of 35 years, the data is limited. One study has followed such young cohort with breast cancer diagnosis for 13 years and 47 women with at least one pregnancy including full-term pregnancies and abortions, after breast cancer therapy were identified (13). Pregnancy was not associated with an increased risk of recurrence or poorer survival but the women with a history of pregnancy tended to have earlier stage disease and more ER negative tumors (13). Several meta-analyses have also reached to the same conclusion that pregnancy subsequent to breast cancer therapy does not impact the recurrence rate or overall survival (14-16). In an attempt to overcome a possible bias of a healthy mother effect a meta-analyses of nine studies comprising 1089 pregnancies and 13051 
matched controls that had taken this in consideration found that a pregnancy occurring at least 10 months after diagnosis may result in a survival benefit (HR $0.5195 \%$ CI: 0.42-0.62) (15). Similar results was found in women undergoing surgery for breast cancer compared to a control group (16). A survival benefit was found in women becoming pregnant compared to those who did not (16). A registry study of BRCA $1 / 2$ mutation carriers has found similar results in this group of women; no adverse effect on survival after pregnancy (17).

The data on risk of breast cancer recurrence after breastfeeding is very limited. Very small studies suggest that lactating women do not exhibit increased risk of relapse but the data contain to small numbers of patients for statistical calculations $(11,18)$.

\section{Effects on fertility by breast cancer treatments}

As the data on pregnancy after breast cancer diagnosis and therapy suggest that this can be safe the question regarding fertility preservation before start of treatment needs to be considered in premenopausal women with early stage breast cancer. This counseling needs to be individual and a risk assessment regarding the patient's risk of recurrence needs to be considered, preferably in a multi disciplinary setting. During the last decade an increased number of women are eligible for adjuvant chemotherapy and in combination with an older age for childbearing an increased number of patients will have these concerns. The risk of chemotherapy induced premature ovarian failure is dependent on the age of the woman and the type of chemotherapy. In women under the age of 40 years the risk of ovarian failure is reported to be between 22$61 \%$ whereas in women above the age of 40 years the risk is increased to $61-97 \%(19,20)$. Different chemotherapy regiments affect the risk of ovarian failure at different rates; CMF for six cycles may induce amenorrhea in $20-75 \%$ of the cases (21), FEC for six cycles $50-64 \%$ (22), AC for four cycles $34 \%$ (21), FAC for six cycles $51 \%$ (23) and TAC for six cycles in $61 \%$ of the women (23). An age nearer the natural age of menopause will increase the risk of ovarian 
failure by chemotherapy (24). In women that continue to menstruated during chemotherapy or in the cases where menstruation returns after completion of chemotherapy the natural menopause will appear at a younger age compared to women with no previous chemotherapy $(25,26)$. Even if menses resume after chemotherapy the ovarian reserve may be diminished and fertility impaired (27). As there a no certain measurements of residual ovarian function at the time of start of adjuvant chemotherapy all premenopausal women eligible for a pregnancy after completion of breast cancer therapy need to be counseled in these issues (28). In addition, the success rate of fertility preserving approaches exhibit better outcome if started before chemotherapy compared to after completed therapy (29). In women becoming pregnant after breast cancer no difference was found in cancer outcome in the group of 25 women subjected to assisted reproductive technology (ART) after breast cancer treatment compared to 180 women that became pregnant spontaneously suggesting that ART may be safe after breast cancer therapy (30).

\section{Fertility preservation in breast cancer patients}

\section{Ovarian suppression with gonadotropin-releasing hormone (GnRH) analogues}

Several randomized trials have been conducted investigating a possible role of temporary ovarian suppression using GnRH analogues for preservation of ovarian function during chemotherapy with disparate results. However, a recent systematic review and meta-analyses of randomized trials found that $\mathrm{GnRH}$ analogues significantly reduce the risk of chemotherapy induced ovarian failure in young cancer patients (31). In addition, a recently published randomized trial of chemotherapy to premenopausal women with ER-negative breast cancer plus minus the addition of the GnRH agonist goserelin showed a significant decreased risk of ovarian failure (OR 0.3; 95\% CI: 0.09-0.97), reduced risk of early menopause, and increased number of pregnancies in the goserelin group (32). These results were in line with previous 
results using another GnRH agonist, triptorelin (33). In women with ER-positive breast cancer there are no safety data of giving ovarian suppression concurrent with chemotherapy. Theoretically, a very fast decrease of estrogen levels as a result of GnRH agonists given before the start of chemotherapy would decrease the proliferation rate of ER-positive breast cancer cells and thus, decrease the efficacy of chemotherapy. In postmenopausal patients it has been evaluated if the anti-estrogen drug tamoxifen given concurrent or sequential with chemotherapy affected disease-free survival and overall survival (34). Borderline significance for increased disease-free survival was found for the sequential group whereas no difference was found for overall survival (34). Concurrent anti-estrogen therapy in ER-positive breast cancer is therefore not recommended today.

In the recently reported TEXT trial a subgroup of premenopausal women with ER-positive breast cancer received GnRH agonist concurrently with chemotherapy in addition to oral tamoxifen or an aromatase inhibitor starting after the completion of chemotherapy (35). Although an excellent survival was reported in all groups these women received an additional of five years of anti-estrogen therapy after the completion chemotherapy (35). These results cannot be extrapolated to a short term ovarian suppression during chemotherapy followed by pregnancy and caution is therefore recommended for giving GnRH agonists concomitant to chemotherapy in women with ER-positive breast cancer.

\section{Embryo and oocyte cryopreservation}

Embryo and oocyte cryopreservation are established infertility treatments. Embryo cryopreservation has excellent results but has disadvantage of the requirement of a partner or sperm donor. Both embryo and oocyte cryopreservation requires ovarian stimulation and oocyte retrieval and may result in a relative delay of the start of chemotherapy and an increased level of estrogen during stimulation. A delay of the start of chemotherapy can be avoided by using random stimulation protocols as these have been found to be as effective as conventional 
protocols (36). In addition, protocols using tamoxifen or the aromatase inhibitor letrozol have been shown to give an adequate yield of oocytes and decreased estrogen levels compared to standard stimulation protocols $(37,38)$. As tamoxifen may cause congenital abnormalities stimulations with aromatase inhibitors should be preferred (39). In one study with a 2-year follow-up, these protocols seem safe for women with ER-positive breast cancer (40) but longterm data on safety are still missing. In a very small study of women subjected to two cycles of stimulation $(n=17)$ compared to one cycle $(n=61)$ no difference in recurrence rate between the groups was found (41).

\section{Ovarian tissue cryopreservation}

Restoration of ovarian tissue function after re-transplantation of cryopreserved ovarian tissue is a rapidly developing technique. During the last years reports of live births after such fertility preservation approach has increased and to date more than 20 women is reported to have given birth after ovarian tissue transplantation including a woman exposed to pelvic radiotherapy (42, 43). Despite the fast development of these techniques they are still considered to be experimental (44). Another issue regarding this approach to take into consideration is the potential risk of re-transplantation of cancer cells. Even though random sections of cryopreserved ovarian tissue can be checked for cancer cells the actual tissue that will be retransplanted into the woman cannot be evaluated for possible residual disease.

The ovary may be a metastatic site for many cancer types (45). The prevalence of ovarian metastasis or micro-metastasis to the ovaries by breast cancer is unknown. In a series of women subjected to surgery for metastatic lesions to the ovaries, approximately $10-20 \%$ had primary tumors originating in from the breast (46-48). Lobular carcinomas of the breast may spread to the pelvic cavity including the ovaries with a higher frequency compared to ductal carcinomas of the breast $(49,50)$. Therefore, in women with lobular carcinomas caution may be warranted 
regarding ovarian tissue cryopreservation and in women with BRCA1 and BRCA 2 mutations with increase risk of ovarian cancer ovarian tissue cryopreservation are not suitable at any time.

\section{Conclusions}

Currently available data suggest that the risk of recurrence or death from breast cancer do not increase by a pregnancy after the completion of adjuvant breast cancer therapy. Although there is a lack of high-level evidence, as no randomized trials have been conducted because of feasibility reasons, there are no reasons for proscription against pregnancy for affected women. Multidisciplinary counseling with specialist with expertise in different areas such as surgery, pathology, radiology, and oncology is now considered as standard in modern breast cancer management. In young women the possibility of infertility by the cancer treatment, the possibilities of fertility preservation, and the risk of a subsequent pregnancy needs to be addressed in collaboration with a specialist of reproductive gynecology before the start of chemotherapy in all eligible women (51). The woman's individual risk needs to be assessed in every case. In addition to the lack of high-level evidence regarding pregnancy after breast cancer treatment per se data regarding the safety of one or several cycles of hormonal preparation of the endometrium before transfer of frozen embryos or fertilized frozen oocytes are lacking. This needs to be addressed in future studies. Another issue is women with ERpositive breast cancer that are prescribed anti-estrogen therapy for 5-10 years, which will significantly reduce their chance of conception. The safety of a shorter duration of this therapy for an up to 2 years interruption for pregnancy attempts and thereafter a resumption of the antiestrogen therapy for full duration is been shown to be feasible and is underway (NCT02308085, ClinicalTrials.gov) (52). The result of that trial will be very important for the counseling of this large group of women. 
Declaration of interest: The author declares no competing interests. 


\section{References}

1. Statistics - Health and Medical Care. Cancer Incidence in Sweden 20132014.

Available from: http://www.socialstyrelsen.se/publikationer2014/2014-12-10.

2. NORDCAN. 2015. Available from: http://www-

dep.iarc.fr/NORDCAN/SW/StatsFact.asp?cancer=200\&country=0.

3. Partridge AH, Gelber S, Peppercorn J, Sampson E, Knudsen K, Laufer M, et al. Web-based survey of fertility issues in young women with breast cancer. Journal of clinical oncology : official journal of the American Society of Clinical Oncology. 2004;22(20):417483.

4. Rippy EE, Karat IF, Kissin MW. Pregnancy after breast cancer: the importance of active counselling and planning. Breast. 2009;18(6):345-50.

5. Azim HA, Jr., Santoro L, Russell-Edu W, Pentheroudakis G, Pavlidis N, Peccatori FA. Prognosis of pregnancy-associated breast cancer: a meta-analysis of 30 studies. Cancer treatment reviews. 2012;38(7):834-42.

6. Amant F, von Minckwitz G, Han SN, Bontenbal M, Ring AE, Giermek J, et al. Prognosis of women with primary breast cancer diagnosed during pregnancy: results from an international collaborative study. Journal of clinical oncology : official journal of the American Society of Clinical Oncology. 2013;31(20):2532-9.

7. Litton JK, Warneke CL, Hahn KM, Palla SL, Kuerer HM, Perkins GH, et al. Case control study of women treated with chemotherapy for breast cancer during pregnancy as compared with nonpregnant patients with breast cancer. The oncologist. 2013;18(4):36976.

8. Mueller BA, Simon MS, Deapen D, Kamineni A, Malone KE, Daling JR.

Childbearing and survival after breast carcinoma in young women. Cancer. 2003;98(6):113140. 
The biological features and prognosis of breast cancer diagnosed during pregnancy: a casecontrol study. Acta oncologica. 2012;51(5):653-61.

10. Sankila R, Heinavaara S, Hakulinen T. Survival of breast cancer patients after subsequent term pregnancy: "healthy mother effect". American journal of obstetrics and gynecology. 1994;170(3):818-23.

11. Azim HA, Jr., Kroman N, Paesmans M, Gelber S, Rotmensz N, Ameye L, et al. Prognostic impact of pregnancy after breast cancer according to estrogen receptor status: a multicenter retrospective study. Journal of clinical oncology : official journal of the American Society of Clinical Oncology. 2013;31(1):73-9.

12. Kroman N, Jensen MB, Wohlfahrt J, Ejlertsen B, Danish Breast Cancer Cooperative G. Pregnancy after treatment of breast cancer--a population-based study on behalf of Danish Breast Cancer Cooperative Group. Acta oncologica. 2008;47(4):545-9. 13. Blakely LJ, Buzdar AU, Lozada JA, Shullaih SA, Hoy E, Smith TL, et al. Effects of pregnancy after treatment for breast carcinoma on survival and risk of recurrence. Cancer. 2004;100(3):465-9.

14. Azim HA, Jr., Santoro L, Pavlidis N, Gelber S, Kroman N, Azim H, et al. Safety of pregnancy following breast cancer diagnosis: a meta-analysis of 14 studies. European journal of cancer. 2011;47(1):74-83.

15. Valachis A, Tsali L, Pesce LL, Polyzos NP, Dimitriadis C, Tsalis K, et al. Safety of pregnancy after primary breast carcinoma in young women: a meta-analysis to overcome bias of healthy mother effect studies. Obstetrical \& gynecological survey. 2010;65(12):786-93. 
16.

Luo M, Zeng J, Li F, He L, Li T. Safety of pregnancy after surgical treatment for breast cancer: a meta-analysis. International journal of gynecological cancer : official journal of the International Gynecological Cancer Society. 2014;24(8):1366-72.

17. Valentini A, Lubinski J, Byrski T, Ghadirian P, Moller P, Lynch HT, et al. The impact of pregnancy on breast cancer survival in women who carry a BRCA1 or BRCA2 mutation. Breast Cancer Res Treat. 2013;142(1):177-85.

18. Azim HA, Jr., Bellettini G, Liptrott SJ, Armeni ME, Dell'Acqua V, Torti F, et al. Breastfeeding in breast cancer survivors: pattern, behaviour and effect on breast cancer outcome. Breast. 2010;19(6):527-31.

19. Del Mastro L, Venturini M, Sertoli MR, Rosso R. Amenorrhea induced by adjuvant chemotherapy in early breast cancer patients: prognostic role and clinical implications. Breast Cancer Res Treat. 1997;43(2):183-90.

20. Simon B, Lee SJ, Partridge AH, Runowicz CD. Preserving fertility after cancer. CA Cancer J Clin. 2005;55(4):211-28; quiz 63-4.

21. Bines J, Oleske DM, Cobleigh MA. Ovarian function in premenopausal women treated with adjuvant chemotherapy for breast cancer. J Clin Oncol. 1996;14(5):1718-29. 22. Venturini M, Del Mastro L, Aitini E, Baldini E, Caroti C, Contu A, et al. Dosedense adjuvant chemotherapy in early breast cancer patients: results from a randomized trial. $\mathbf{J}$ Natl Cancer Inst. 2005;97(23):1724-33.

23. Martin M, Pienkowski T, Mackey J, Pawlicki M, Guastalla JP, Weaver C, et al. Adjuvant docetaxel for node-positive breast cancer. N Engl J Med. 2005;352(22):2302-13. 24. Goodwin PJ, Ennis M, Pritchard KI, Trudeau M, Hood N. Risk of menopause during the first year after breast cancer diagnosis. Journal of clinical oncology : official journal of the American Society of Clinical Oncology. 1999;17(8):2365-70. 
al. Incidence and time course of bleeding after long-term amenorrhea after breast cancer treatment: a prospective study. Cancer. 2010;116(13):3102-11.

26. Partridge A, Gelber S, Gelber RD, Castiglione-Gertsch M, Goldhirsch A, Winer E. Age of menopause among women who remain premenopausal following treatment for early breast cancer: long-term results from International Breast Cancer Study Group Trials V and VI. European journal of cancer. 2007;43(11):1646-53.

27. Partridge AH, Ruddy KJ, Gelber S, Schapira L, Abusief M, Meyer M, et al. Ovarian reserve in women who remain premenopausal after chemotherapy for early stage breast cancer. Fertility and sterility. 2010;94(2):638-44.

28. Partridge AH, Pagani O, Abulkhair O, Aebi S, Amant F, Azim HA, Jr., et al. First international consensus guidelines for breast cancer in young women (BCY1). Breast. 2014;23(3):209-20.

29. Dolmans MM, Demylle D, Martinez-Madrid B, Donnez J. Efficacy of in vitro fertilization after chemotherapy. Fertil Steril. 2005;83(4):897-901.

30. Goldrat O, Kroman N, Peccatori FA, Cordoba O, Pistilli B, Lidegaard O, et al. Pregnancy following breast cancer using assisted reproduction and its effect on long-term outcome. European journal of cancer. 2015;51(12):1490-6.

31. Del Mastro L, Ceppi M, Poggio F, Bighin C, Peccatori F, Demeestere I, et al. Gonadotropin-releasing hormone analogues for the prevention of chemotherapy-induced premature ovarian failure in cancer women: systematic review and meta-analysis of randomized trials. Cancer treatment reviews. 2014;40(5):675-83.

32. Moore HC, Unger JM, Phillips KA, Boyle F, Hitre E, Porter D, et al. Goserelin for ovarian protection during breast-cancer adjuvant chemotherapy. The New England journal of medicine. 2015;372(10):923-32. 
of the gonadotropin-releasing hormone analogue triptorelin on the occurrence of chemotherapy-induced early menopause in premenopausal women with breast cancer: a randomized trial. JAMA. 2011;306(3):269-76.

34.

Albain KS, Barlow WE, Ravdin PM, Farrar WB, Burton GV, Ketchel SJ, et al. Adjuvant chemotherapy and timing of tamoxifen in postmenopausal patients with endocrineresponsive, node-positive breast cancer: a phase 3, open-label, randomised controlled trial. Lancet. 2009;374(9707):2055-63.

35. Pagani O, Regan MM, Walley BA, Fleming GF, Colleoni M, Lang I, et al. Adjuvant exemestane with ovarian suppression in premenopausal breast cancer. The New England journal of medicine. 2014;371(2):107-18.

36. Cakmak H, Katz A, Cedars MI, Rosen MP. Effective method for emergency fertility preservation: random-start controlled ovarian stimulation. Fertility and sterility. 2013;100(6):1673-80.

37. Oktay K, Buyuk E, Libertella N, Akar M, Rosenwaks Z. Fertility preservation in breast cancer patients: a prospective controlled comparison of ovarian stimulation with tamoxifen and letrozole for embryo cryopreservation. Journal of clinical oncology : official journal of the American Society of Clinical Oncology. 2005;23(19):4347-53.

38. Oktay K, Hourvitz A, Sahin G, Oktem O, Safro B, Cil A, et al. Letrozole reduces estrogen and gonadotropin exposure in women with breast cancer undergoing ovarian stimulation before chemotherapy. The Journal of clinical endocrinology and metabolism. 2006;91(10):3885-90.

39. Braems G, Denys H, De Wever O, Cocquyt V, Van den Broecke R. Use of tamoxifen before and during pregnancy. The oncologist. 2011;16(11):1547-51. 
40. Azim AA, Costantini-Ferrando M, Oktay K. Safety of fertility preservation by ovarian stimulation with letrozole and gonadotropins in patients with breast cancer: a prospective controlled study. Journal of clinical oncology : official journal of the American Society of Clinical Oncology. 2008;26(16):2630-5.

41. Turan V, Bedoschi G, Moy F, Oktay K. Safety and feasibility of performing two consecutive ovarian stimulation cycles with the use of letrozole-gonadotropin protocol for fertility preservation in breast cancer patients. Fertility and sterility. 2013;100(6):1681-5 e1. 42. Donnez J, Dolmans MM, Pellicer A, Diaz-Garcia C, Sanchez Serrano M, Schmidt KT, et al. Restoration of ovarian activity and pregnancy after transplantation of cryopreserved ovarian tissue: a review of 60 cases of reimplantation. Fertility and sterility. 2013;99(6):1503-13.

43. Rodriguez-Wallberg KA, Karlstrom PO, Rezapour M, Castellanos E, Hreinsson J, Rasmussen C, et al. Full-term newborn after repeated ovarian tissue transplants in a patient treated for Ewing sarcoma by sterilizing pelvic irradiation and chemotherapy. Acta obstetricia et gynecologica Scandinavica. 2015;94(3):324-8.

44. Practice Committee of American Society for Reproductive M. Ovarian tissue cryopreservation: a committee opinion. Fertility and sterility. 2014;101(5):1237-43.

45. Kiyokawa T, Young RH, Scully RE. Krukenberg tumors of the ovary: a clinicopathologic analysis of 120 cases with emphasis on their variable pathologic manifestations. The American journal of surgical pathology. 2006;30(3):277-99. 46. Moore RG, Chung M, Granai CO, Gajewski W, Steinhoff MM. Incidence of metastasis to the ovaries from nongenital tract primary tumors. Gynecologic oncology. 2004;93(1):87-91. 
47.

Ayhan A, Guvenal T, Salman MC, Ozyuncu O, Sakinci M, Basaran M. The role of cytoreductive surgery in nongenital cancers metastatic to the ovaries. Gynecologic oncology. 2005;98(2):235-41.

48. Curtin JP, Barakat RR, Hoskins WJ. Ovarian disease in women with breast cancer. Obstetrics and gynecology. 1994;84(3):449-52.

49. Harris M, Howell A, Chrissohou M, Swindell RI, Hudson M, Sellwood RA. A comparison of the metastatic pattern of infiltrating lobular carcinoma and infiltrating duct carcinoma of the breast. British journal of cancer. 1984;50(1):23-30.

50. Lamovec J, Bracko M. Metastatic pattern of infiltrating lobular carcinoma of the breast: an autopsy study. Journal of surgical oncology. 1991;48(1):28-33.

51. Loren AW, Mangu PB, Beck LN, Brennan L, Magdalinski AJ, Partridge AH, et al. Fertility preservation for patients with cancer: American Society of Clinical Oncology clinical practice guideline update. Journal of clinical oncology : official journal of the American Society of Clinical Oncology. 2013;31(19):2500-10.

52. Pagani O, Ruggeri M, Manunta S, Saunders C, Peccatori F, Cardoso F, et al. Pregnancy after breast cancer: Are young patients willing to participate in clinical studies? Breast. 2015;24(3):201-7. 